\title{
Tumores que comprometen la rinofaringe, nuestra experiencia
}

\author{
Tumors of rhinopharynx. Our experience
}

Roberto Arias $A^{1,2}$, Mariana Bogado $C^{3}$, Homero Sariego $\mathbf{R}^{1}$.

\begin{abstract}
RESUMEN
Las lesiones que comprometen la rinofaringe son de difícil diagnóstico debido al complejo acceso a esta región, excepto cuando éstas alcanzan mayor tamaño o cuando presentan síntomas y/o signos secundarios al compromiso de estructuras adyacentes 0 de diseminación. El propósito de esta investigación fue analizar y describir casos clínicos de pacientes portadores de patología tumoral de la rinofaringe, con la finalidad de aportar nuevos antecedentes en un área en la cual existen pocos estudios publicados, dada su baja incidencia. Se realizó un análisis descriptivo de corte transversal de 10 casos clínicos de pacientes portadores de patologías que comprometían la rinofaringe, evaluados en la unidad de Otoneurología del Instituto de Neurocirugía (1999-2008). De los casos analizados, se observó una mediana de edad de 52,5 años, el tiempo desde la presentación de los síntomas hasta el diagnóstico fue de 8,5 meses. Los tumores de origen epitelial fueron la causa más frecuente, presentándose además tumores de origen óseo y linfoide. El tratamiento fue quirúrgico y asociado a terapia complementaria según necesidad. El número de casos analizados es pequeño, por lo que no es posible sacar conclusiones, sin embargo, dado la clínica inespecífica es fundamental un alto índice de sospecha, e idealmente en el estudio inicial realizar un examen endoscópico en forma rutinaria.
\end{abstract}

Palabras clave: Rinofaringe, Tumor.

\begin{abstract}
Lesions of rhinopharynx are difficult to diagnose due to the complex access to this area, except when they reach a greater size or when they present symptoms and/or signs secondary to the compression of adjacent structures or dissemination. The aim of this study was to analyze and to describe clinical cases of patients with rhinopharynx pathologies, with the purpose of giving new antecedents in an area where are few published studies, due to its low incidence. A cross-sectional descriptive study was made of 10 clinical cases of patients with rhinopharynx tumors, which were evaluated in
\end{abstract}

\footnotetext{
1 Médico de la Unidad de Otoneurología, Instituto de Neurocirugía e Investigaciones Cerebrales Alfonso Asenjo, Santiago.

2 Médico del Servicio de Otorrinolaringología del Hospital Salvador, Santiago

3 Médico Cirujano.
} 
the unit of Otoneurology of the Neurosurgery Institute (1999-2008). The median age was 52.5 years, and the time since the symptoms appear and the diagnosis was made was of 8.5 months. The most frequent causes were tumors of epithelial origin, but also osseous and lymphoid tumors were observed. Treatment was surgical and/or associated to complementary therapy. Due to the small number cases analyzed it is not possible to draw conclusions, nevertheless, it is important to have a high index of suspicion, given the unspecific symptom and signs that they may present, and ideally in the initial study of these patients it is necessary to perform an endoscopic evaluation.

Key words: Rhinopharynx, Tumor.

\section{INTRODUCCIÓN}

La rinofaringe 0 nasofaringe ${ }^{1}$ es una cavidad aérea cuboidea que corresponde a la porción superior de la faringe, se ubica superior al paladar blando y representa una prolongación posterior de las cavidades nasales ${ }^{2}$, limita posteriormente con la apófisis basilar del hueso occipital y del axis y cranealmente con el cuerpo del esfenoides ${ }^{3}$. La rinofaringe está tapizada por epitelio cilíndrico, el que se reconocen células caliciformes (secretoras), además posee epitelio escamoso en parches (metaplasia). Bajo el epitelio existe abundante tejido linfoide en la submucosa (tejido linfoide asociado a Mucosas - MALT) $)^{3-5}$.

Debido al complejo acceso al rinofarinx, las lesiones que se ubican en esta región son de difícil diagnóstico, excepto cuando alcanzan mayor tamaño, produciendo obstrucción nasal 0 epistaxis, 0 cuando presentan síntomas y signos secundarios al compromiso de las estructuras adyacentes 0 de diseminación por vía linfática a ganglios cervicales. Razón por la cual, las diversas patologías que afectan esta región son diagnosticadas en forma tardía u ocasionalmente en forma incidental a través de exámenes radiológicos ${ }^{4}$. Además debido a la diversa histología de la región, el diagnóstico diferencial de las lesiones que pueden presentarse en esta zona es variado ${ }^{6}$.

El conocimiento clínico y el advenimiento de las técnicas modernas de radiología, tales como la tomografía computarizada (TC), resonancia magnética (RM), angiografía, angio-tomografía computarizada (Angio-TC) y la tomografía por emi- sión de positrones (PET), junto a la incorporación de la exploración endoscópica, han permitido mayor exhaustividad en la búsqueda de estas patologías, permitiendo así, pesquisar estas lesiones en forma más precoz.

Los tumores que comprometen la rinofaringe pueden ser de origen epitelial, conjuntivo, linfoide o glandular. Los tumores más frecuentes e importantes son: el angiofibroma, el carcinoma nasofaringeo y el linfoma, sin embargo, también es posible la aparición en esta región de metástasis de tumor primario de mama, renales o melanomas malignos, además puede existir compromiso de la región por vecindad de tumores de áreas adyacentes, ya sea de origen vascular, cerebral, o de senos paranasales. Por otro lado, también pueden producirse lesiones no tumorales; tanto granulomatosas como infecciosas ${ }^{6,7}$.

Los tumores de la rinofaringe son poco frecuentes y tanto su incidencia, como su distribución por edad y sexo dependen, del tipo histológico de la lesión. El tumor benigno más frecuente es el angiofibroma nasofaringeo juvenil, el cual representa el $0,05 \%$ de los tumores de cabeza y cuello ${ }^{8}$, se manifiesta exclusivamente en hombres, con un rango etario de 7 a 19 años, siendo raro después de los 25 años $^{6,8}$. Los tumores malignos de la nasofaringe son poco frecuentes ${ }^{7}$, reportándose una incidencia de 0,5 a 2 casos por 100.000 habitantes al año en Europa y Estados Unidos ${ }^{9}$. En Chile no existen estadísticas de su incidencia, sólo se dispone de cifras nacionales de mortalidad, sin embargo, el año 2006 sólo hubo 4 muertes por esta causa a nivel nacional ${ }^{10} \mathrm{y}$ entre los años 1991 
y 1999 correspondió al 0,7\% de las muertes de causa otorrinolaringológica ${ }^{11}$. El carcinoma de rinofarinx es el tumor maligno más frecuente que compromete esta región, con una incidencia estimada en la población general menor al 1 por 100.000 habitantes al año en países como Estados Unidos y Europa, alcanzando una mayor incidencia en países como China y en el Sudeste Asiático, donde se describe que la tasa es de 20 por 100.000 personas al año ${ }^{12}$. Se presenta con mayor frecuencia en hombres, con una razón de 3:1. Por otro lado, los linfomas son los tumores no epiteliales más frecuentes de cabeza y cuello ${ }^{6}$.

Las manifestaciones clínicas de las lesiones de rinofaringe pueden clasificarse de una forma esquemática, de acuerdo a la región comprometida en síntomas:

- Generales: compromiso del estado general y baja de peso.

- Nasal: obstrucción nasal, rinorrea, epistaxis, anosmia y alteraciones de la calidad de la voz.

- Otológicos: sensación de oído tapado, hipoacusia fluctuante, otitis serosa unilateral, otalgia.

- Neurológico: cefalea, neuralgias del V par.

- Orofaringe y faringe: alteración del gusto y la deglución (IX, X, XI, XII pares craneanos bajos).

- Ocular: amaurosis, exoftalmos, diplopia (II, III, IV, VI par craneano).

Como se mencionó en un comienzo, la imagenología ha permitido detectar tumores de la rinofaringe en estadios precoces. Dentro de los estudios que permiten estudiar esta región podemos mencionar: el TC (con contraste), ya que permite determinar si existe compromiso óseo tanto de la zona en cuestión como de estructuras adyacentes, la RM permite complementar el estudio, especialmente si existe compromiso de base de cráneo y de tejidos blandos, además orientar a la etiología de la lesión, ya sea tumoral 0 inflamatoria. La angiografía es de utilidad en el caso de tumores de origen vascular.

Dado que la mayoría de las lesiones que se presentan en esta región son malignas, el pronóstico del paciente depende principalmente del diagnóstico histopatológico de la lesión, el cual puede realizarse mediante cirugía endoscópica primaria- mente, técnica quirúrgica que puede permitir también, reducir la masa tumoral, y excepcionalmente ser curativa por sí sola.

El estudio histopatológico permite determinar el diagnóstico histopatológico definitivo, por lo que debe realizarse una biopsia de la lesión, la cual se puede realizar con anestesia local bajo visión nasofibroscópica o con anestesia general en pabellón.

Para el adecuado estudio de los tumores de rinofaringe, se debe tener un conocimiento acabado de la anatomía de esta zona de tal manera de tener un alto índice de sospecha frente a los signos y/o síntomas mencionados, debido a la escasa sintomatología que presentan inicialmente y a que pueden corresponder a distintos tipos de tumores. Es deber de todo otorrinolaringólogo realizar un examen endoscópico flexible como parte inicial en el estudio de la rinofaringe.

El propósito de esta investigación es analizar y describir casos clínicos de pacientes portadores de patología tumoral que comprometen la rinofaringe, con la finalidad de aportar nuevos antecedentes sobre el tema, que en la actualidad representa un desafío importante para la otorrinolaringología por las causas ya mencionadas, y sobre la cual no hay gran experiencia por su baja incidencia.

\section{OBJETIVO}

Analizar y describir el aspecto nosológico de pacientes portadores de patología rinofaringea, tanto en su evolución diagnóstica, manejo y tratamiento.

\section{MATERIAL Y MÉTODO}

Estudio descriptivo de corte transversal de casos clínicos de pacientes portadores de patologías que comprometen la rinofaringe. El estudio incluye casos evaluados desde el año 1999 a 2008.

Para la realización de esta investigación se revisó los casos clínicos de pacientes evaluados por la unidad de otoneurología del Instituto de Neurocirugía portadores de patologías que comprometen el rinofarinx. La información fue analizada con el programa estadístico SPSS 11.0. 
Los resultados obtenidos en este estudio fueron analizados utilizando cifras absolutas y porcentuales. Para las variables cuantitativas se calculó la mediana como medida de tendencia central. Para las variables cualitativas se calcularon medidas de distribución de frecuencia (cifras absolutas y porcentuales).

\section{RESULTADOS}

Se analizó 10 casos de tumores que comprometieron la rinofaringe, los cuales se resumen en la Tabla 1. Por el escaso número de casos analizados, se analizó la mediana de la edad, la cual corresponde a 52,5 años, con un rango de 18 a 64 años de edad, pero sí se excluye el caso de fibroangioma la mediana es de 57 años, con un rango de 38 a 64 años. Los tumores de rinofarinx no poseen un rango de edad determinado, ya que la edad de presentación depende de la histología del tumor. El $70 \%$ de los casos corresponde a pacientes hombres.

El tiempo diagnóstico representa el tiempo transcurrido entre que el paciente inicia la sintomatología y se le diagnostica un tumor de rinofarinx, la mediana del tiempo diagnóstico fue de 8,5 meses, con un rango de 5 a 24 meses.

Al analizar la sintomatología presentada, se puede observar que los síntomas oculares y neurológicos fueron los más frecuentes ( $30 \%$ cada uno de ellos), los síntomas otológicos se presentaron en $20 \%$ de los casos. Los síntomas generales y nasales fueron menos frecuentes (sólo 10\% de los casos) y no se encontró sintomatología de compromiso de orofarine y faringe.

Al observar el estudio de imágenes se debe mencionar que en $50 \%$ de los casos no se plantea probable etiología, y sólo en $30 \%$ de los casos

Tabla 1. Casos de tumores que comprometen la rinofaringe

\begin{tabular}{|c|c|c|c|c|c|c|}
\hline Caso & $\begin{array}{l}\text { Edad } \\
\text { (años) }\end{array}$ & $\begin{array}{l}\text { Síntoma } \\
\text { predominante }\end{array}$ & $\begin{array}{l}\text { Diagnostico } \\
\text { histológico }\end{array}$ & Tratamiento & $\begin{array}{l}\text { Diagnóstico } \\
\text { radiológico presuntivo }\end{array}$ & $\begin{array}{l}\text { Tiempo diagnóstico } \\
\text { (en meses) }^{*}\end{array}$ \\
\hline 1 & 42 & $\begin{array}{l}\text { Alteración del } \\
\text { estado general }\end{array}$ & Linfoma & $\begin{array}{l}\text { Quimioterapia } \\
\text { Radioterapia }\end{array}$ & No planteado & 12 \\
\hline 2 & 18 & Epistaxis & Fibroangioma & Cirugía & Relacionado & 5 \\
\hline 3 & 62 & $\begin{array}{l}\text { Sensación de oído } \\
\text { tapado }\end{array}$ & $\begin{array}{l}\text { Carcinoma } \\
\text { epidermoide }\end{array}$ & $\begin{array}{l}\text { Quimioterapia } \\
\text { Radioterapia/QX }\end{array}$ & Relacionado & 7 \\
\hline 4 & 58 & Cefalea & Rabdomiosarcoma & $\begin{array}{l}\text { Quimioterapia } \\
\text { Radioterapia }\end{array}$ & No planteado & 8 \\
\hline 5 & 57 & $\begin{array}{l}\text { Neuralgia V par } \\
\text { e hipoestesia facial }\end{array}$ & $\begin{array}{l}\text { Carcinoma } \\
\text { adenoideo quístico }\end{array}$ & $\begin{array}{l}\text { Quimioterapia } \\
\text { Radioterapia }\end{array}$ & Sin relación & 18 \\
\hline 6 & 48 & Fasciculaciones linguales & Meningioma & Cirugía & Relacionado & 6 \\
\hline 7 & 63 & Exoftalmos & $\begin{array}{l}\text { Carcinoma } \\
\text { adenoideo quístico }\end{array}$ & $\begin{array}{l}\text { Quimioterapia } \\
\text { Radioterapia }\end{array}$ & No planteado & 24 \\
\hline 8 & 64 & Diplopía & $\begin{array}{l}\text { Carcinoma } \\
\text { epidermoide }\end{array}$ & $\begin{array}{l}\text { Quimioterapia } \\
\text { Radioterapia } \\
\text { Cirugía }\end{array}$ & Sin relación & 8 \\
\hline 9 & 38 & Diplopía & Cordoma & Cirugía & No planteado & 9 \\
\hline 10 & 47 & $\begin{array}{l}\text { Sensación de oído } \\
\text { tapado }\end{array}$ & $\begin{array}{l}\text { Carcinoma } \\
\text { epidermoide }\end{array}$ & $\begin{array}{l}\text { Quimioterapia } \\
\text { Radioterapia }\end{array}$ & No planteado & 12 \\
\hline
\end{tabular}

*Tiempo diagnóstico: tiempo transcurrido entre la aparición de los síntomas y el diagnóstico de tumor de rinofaringe. 
hubo cierto grado de concordancia entre el diagnóstico planteado por radiología y la biopsia realizada.

El diagnóstico histológico más frecuente fueron los tumores de origen epitelial (carcinoma) en $50 \%$ de los casos, además se encontró tumores de origen óseo, linfoide y de anexos.

El tratamiento fue quirúrgico en la mitad de los casos $(50 \%)$, siendo necesaria la utilización de terapia complementaria coadyuvante en 7 de ellos, radioterapia más quimioterapia.

\section{DISCUSIÓN}

Las lesiones tumorales que comprometen el rinofarínx son poco frecuentes y requieren un alto índice de sospecha, ya que su sintomatología suele presentarse en forma tardía, ya sea por efecto de masa (lo que puede producir obstrucción nasal y epistaxis), por síntomas y signos secundarios al compromiso de las estructuras adyacentes, por diseminación por vía linfática a ganglios cervicales o por ser diagnosticados como hallazgo mediante estudios imagenológicos.

Dado que el número de casos analizados en este estudio es pequeño, no es posible sacar conclusiones sobre esta patología en esta serie, sin embargo permite observar que en algunos casos la sintomatología presentada fue inespecífica (sensación de oído tapado y cefalea), o con sintomatología que posiblemente consultó en otras especialidades inicialmente (diplopia, exoftalmo, fasciculaciones linguales, cefalea, neuralgia al trigémino, compromiso del estado general), de manera que es fundamental, como ya se mencionó, tener un alto índice de sospecha, idealmente en el estudio otorrinolaringólogo, debiese realizarse un examen endoscópico en forma rutinaria, como parte inicial en el estudio de la rinofaringe.

\section{BIBLIOGRAFÍA}

1. National Library of Medicine - Medical Subject Headings, [en línea]. U.S. National Library of Medicine, National Institutes of Health, 2009.
En: http://www.nlm.nih.gov/cgi/mesh/2009/ MB_cgi, Citado: 20 de Mayo, 2009.

2. Moore K. El Cuello. En: Anatomía con orientación Clínica, Moore, K. Tercera Edición. Editorial Panamericana. 1993, Págs. 854-63.

3. Rojas S, Rodríguez R, Ruiz G, Rubio B. Epidemiología descriptiva de los tumores de la nasofaringe en cuatro grandes hospitales públicos de la comunidad de Madrid. Departamento de Medicina Preventiva y Salud Pública e Historia de la Ciencia, Universidad Complutense de Madrid. En línea en: http:// www.ucm.es/info/dosis/Preventiva/jor_xiv/ Mesa4.21.pdf. Citado: 20 de mayo, 2009.

4. Stevens A, Lowe J. Chapter 12: Oral and ENT Pathology. En: Stevens A, Lowe J. Pathology. $2^{\mathrm{a}}$ Edición. Editorial Elsevier Health Sciences. 2000, United States. Pág. 236.

5. Stevens A, Lowe J. Histología Humana. 3a Edición. España. 2006. Editorial Elsevier España. Pág. 168.

6. Ximena Montero P, Lisbeth Platzer M, Marcela Castillo F. Actualizaciones en tumores de rinofaringe. Rev Otorrinolaringol Cir Cabeza Cuello 2005; 65: 221-32.

7. Shanmugaratnam K, Sobin L. The World Health Organization histological classification of tumors of the upper respiratory tract and ear. A commentary on the second edition. Cancer 1993; 71(8): 2689-97.

8. Tewfik T, Al Garni M. Juvenile Nasopharyngeal Angiofibroma, [en línea]. E-medicine. En: http:/ /emedicine.medscape.com/article/872580overview, citado: 20 de mayo, 2009.

9. Gordon G, Brockstein B. Epidemiology, etiology, pathology, clinical presentation, and staging of nasopharyngeal carcinoma, en [en línea] en UpToDate. En: http://www.uptodate.c $0 \mathrm{~m} / \mathrm{p}$ a t i e n t s/content/ topic.do?topicKey $=\sim U \mathrm{~kb} x \mathrm{GhqwXWqmsH}$, citado: 25 de mayo, 2009.

10. Departamento de Estadísticas (DEIS), Ministerio de Salud, Chile. Base de datos, defunciones y nacimientos 1990-2006 [en línea]. En: deis.minsal.cl, citado: 25 de mayo, 2009.

11. Bejar M, Cevo J, Romero M, Iñiguez R. Mortalidad nacional en otorrinolaringología. Rev Otorrinolaringol Cir Cabeza Cuello 
[online] 2007; 67(1): 31-7. Citado: 25 de Mayo, 2009.

12. Sun L, Epplein M, Li C, Vaughan T, Weiss N. Trends in the incidence rates of nasopharyngeal carci- noma among Chinese Americans living in Los Angeles County and the San Francisco metropolitan area, 1992-2002. Am J Epidemiol 2005; 162(12): 1174-8. 